\title{
RELATIONSHIP OF ELECTRICALLY INDUCED PAIN TO THE AMPERAGE AND THE WATTAGE OF SHOCK STIMULI
}

\author{
BY HARRIS E. HILL, HAROLD G. FLANARY, CONAN H. KORNETSKY, AND \\ ABRAHAM WIKLER
}

\author{
(From the National Institute of Mental Health, Addiction Research Center, Public Health \\ Service Hospital, Lexington, $K y$.)
}

(Submitted for publication January 10, 1952; accepted March 17, 1952)

\section{INTRODUCTION}

The present paper deals with the development of an apparatus for delivering controlled electric stimuli, and with an attempt to discover which physical aspect of 60 cycle alternating current (voltage, amperage, or wattage) is most closely related to discrimination of shock stimuli in psychological experiments. ${ }^{1}$ Shock, of course, has been the most widely used, and the most convenient type of noxious stimulus for many psychological studies. However, "convenient" should be italicized for, while shock is usually rather simple to apply, accurate control of the most effective physical aspect of this stimulus is difficult.

A survey of the literature indicates that control of voltage alone in psychological studies is quite unsatisfactory. This is frequently the case even when considerable physical resistance is added to the circuit in series with the biological materials.

Problems relating to the use of electric shock stimuli were considered in a round-table discussion of the American Psychological Association $(2,3)$. Although there was no close agreement among members, the general opinion seemed to be that amperage was the physical aspect of shock which should be controlled. Muenzinger and Walz (4) developed a method for controlling D.C. current (to a great extent) despite variations in skin resistance. This technic has proved quite satisfactory for a number of purposes. However, the few psychophysical experiments that have been performed with control of amperage have yielded disparate results. Thus, Dalziel and Lagen (5) found that the integumental threshold for 60 cycle alternating current was 1.1 milliamperes (ma.), while Forbes and Bernstein (6) found great fluc-

\footnotetext{
1 Ramifications of the obvious fact that the value or function that shock acquires is different for different individuals will not be considered in this paper; cf. Tompkins (1).
}

tuations. In a study using a modified method of limits while controlling wattage, Gilmer (7) found a mean absolute threshold at $0.3 \mathrm{ma}$. at a frequency of 64 cycles per second, and a mean threshold value of $3.0 \mathrm{ma}$. at a frequency of 12,500 cycles. It is known, of course, that differences in electrode materials and differences in their size, shape and location may introduce artifacts; however, it would seem that the discrepancies have been so great that they cannot be accounted for on the basis of electrode error. It is evident, therefore, that the possibility must be considered that power may be the most relevant measure of electric shock stimuli in psychological studies. Such a conclusion was reached by Forbes and Bernstein (6) who stated that "neither voltage nor current alone is a satisfactory index of the sensory effects of shock. The results do suggest that power may be such an index."

Quite uniform and stable power thresholds were obtained by Gilmer in the study mentioned above (7). He used a method of limits in which a milliwatt (mw.) scale was employed for determining limens at nine frequencies that ranged from 64 to 12,500 cycles per second. Thresholds were found to be almost identical for his four subjects, being $30.88 \mathrm{mw}$. at 64 cycles and $392 \mathrm{mw}$. at 12,500 cycles.

In support of the hypothesis that power is the most significant physical aspect of shock, it might be noted that several of the devices used for controlling amperage also reduced variations in power. Certainly Muenzinger and Walz (4) reduced the variation in power as they reduced the error in amperage. Also, it might be pointed out that the introduction of complemental resistance into the stimulating circuit in physiological experiments seems to reduce fluctuations in stimulus power. Thus, Jasper (8), in adding 15,000 ohms in series with the subject during studies on chronaxie, was 
in effect exerting control of the energy of the stimulating circuit.

There are indications, also, that power has been partially controlled in conditioning studies. For instance, in studies on dogs, Kellogg (9) maintained a voltage that was just sufficient to force a flexion reflex of four inches. Kellogg, Pronko, and Headlee also used this method for investigations of drugs (10) wherein they believed it necessary to control the unconditioned response according to a behavioral index of the effectiveness of the shock. Later Spooner and Kellogg (11) extended this type of shock control, on the basis of a reaction index, to the conditioning of the finger withdrawal in human subjects. Inspection of Kellogg's (9) voltage curves suggests that the uniformity of the unconditioned response was achieved through at least partial control of the power of stimuli. This is indicated by the gradual decline in the voltage necessary to elicit the unconditioned response as the experiment progressed. Since it may be assumed that the resistance of the biologic circuit declined progressively after repeated shock stimuli, it is apparent that the voltage required to deliver a constant power would also be reduced concomitantly.

The control of shock stimuli in terms of the physiological reaction which they produce has been employed in other areas of conditioning and in neuropharmacological investigation (Wikler [12]). However, it seems to be somewhat inadequate for human studies. One of the reasons is that the frames of reference are different. Extrapolation from results obtained in studies where a reaction was forced by shock, to results obtained in human discrimination of the intensities of stimuli, would seem unwarranted. It would be desirable, therefore, to discover the relative effectiveness of amperage and wattage in representative experimental situations; $e . g$. , in purely physiological studies, in conditioning studies, in discrimination studies and in any other situations displaying unique characteristics. It may be that the effective electrical index in one will be found to be the effective index in all others; this remains to be tested.

Our own pilot work was begun with a modified method of single stimuli using a voltage scale. Standard stimuli were applied one hour previous to the administration of six test series of 11 stimuli each. The subject reported verbally his judg- ment of whether the variable shocks were "stronger" or "weaker" than the previously given standard. Both delivered voltage and amperage were recorded for each stimulation. Results, computed separately on the basis of delivered voltage, amperage and power, showed that voltage was the least significant characteristic; also these computations gave strong indications, but did not prove, that power was the most important physical characteristic affecting judgments.

This work showed one thing quite conclusively. If experiments of any precision were to be undertaken using shock stimuli, a method must be developed for accurately predicting the voltage necessary for producing desired amperages or wattages once the skin resistance was known. A direct application of Ohm's law could not be relied upon in this situation since it is known that capacitance is a factor of some magnitude which must be taken into account in studies on biological circuits (Curtis [13]), especially when skin impedance is relatively high. Therefore, for present purposes it was necessary to devise an apparatus which could be used to deliver shock stimuli of known wattage or amperage, and to construct empirical power curves for the purpose of pre-setting the stimulator for desired shock intensities. After this was accomplished, a procedure was developed to determine the relationship of induced pain to the wattage and amperage of electric shock stimuli.

\section{METHODS}

Apparatus. The biologic resistance of the subject before and after stimulation was measured by use of a General Radio A.C. bridge (Type $650 \mathrm{~A}$ ). In making such measurements the capacitance of the subject was balanced out by adding the required capacitance to the opposite arm of the Wheatstone bridge. A cathode ray oscilloscope was used to determine the state of balance in the bridge circuit.

The circuit of the 60 cycle A.C. stimulating apparatus is illustrated in Figure 1. Essentially, this consists of two parts: (1) a stimulating circuit which permits recording of impressed voltage and actually delivered amperage, and (2) a trigger and relay system which limits the duration of the stimulus to exactly 0.1 second and prevents accidental repetitive shocking of the subject during a 30-second period after each stimulation.

The stimulating circuit is composed of a line source variac and transformer which permits delivery of $0-600$ volts $A . C$. to the subject through an external resistance of 10,000 ohms. The latter serves to minimize the effects of variations in the subject's impedance during passage 
of the stimulating current. Root-mean-square amperage of the stimulating current actually delivered to the subject is measured by a separate circuit (insert, Figure 1). Flow of current in this circuit is induced by that passing through the subject during stimulation. The A.C. induced current is converted by a rectifier into D.C. surges which charge a series of condensors. The resulting voltage drop across one of the condensors, as measured by a standard D.C. vacuum tube voltmeter, is therefore proportional to the A.C. current passing through the subject.

The trigger and relay system limits the stimulating current to a duration of 0.1 second by a circuit consisting of Relay \#1, resistor $R_{1}$, condensor $C_{1}$, and thyratron tube $2 D 21$ and a power source $P$. As long as contact $B$ is closed, current flows through this circuit, Relay \#1 remains activated and contacts $A$ and $D$ remain open. When contact $B$ is opened, this circuit is broken, thereby permitting contacts $A$ and $D$ to close and the stimulating current to pass through the subject. The opening and closing of contact $B$ is almost instantaneous (see below). Hence, the circuit is restored almost immediately after stimulation is begun. However, contact $A$ remains closed until Relay \#1 is reactivated. This interval ( 0.1 second) is determined by the characteristics of thyratron tube $2 D 21, R_{1}$, and $C_{1}$, since immediately after the opening of contact $B$, condensor $C_{1}$ discharges and when its potential drops to about zero, the thyratron tube is fired and Relay \#1 is reactivated, thereby opening contacts $A$ and $D$. Prevention of accidental repetitive shocking of the subject for a period of 30 seconds is insured by the circuit consisting of switch $S_{1}$, condensor $C_{2}$, resistor $R_{2}$, and Relay \#2. When switch $S_{1}$ is closed (by the subject or the experimenter), condensor $C_{2}$ discharges, thereby activating Relay \#2 and opening contact $B$ for a very brief period (approximately $10 \mathrm{msc}$.). The values of $C_{2}$ and $R_{2}$ are such that 30 seconds are required for recharging $C_{2}$ to a voltage which is high enough again to activate Relay \#2.

Subjects. All subjects used in this investigation were male prisoner patients with previous histories of drug addiction ("post-addicts") who had not received drugs for several months prior to the present study, and who

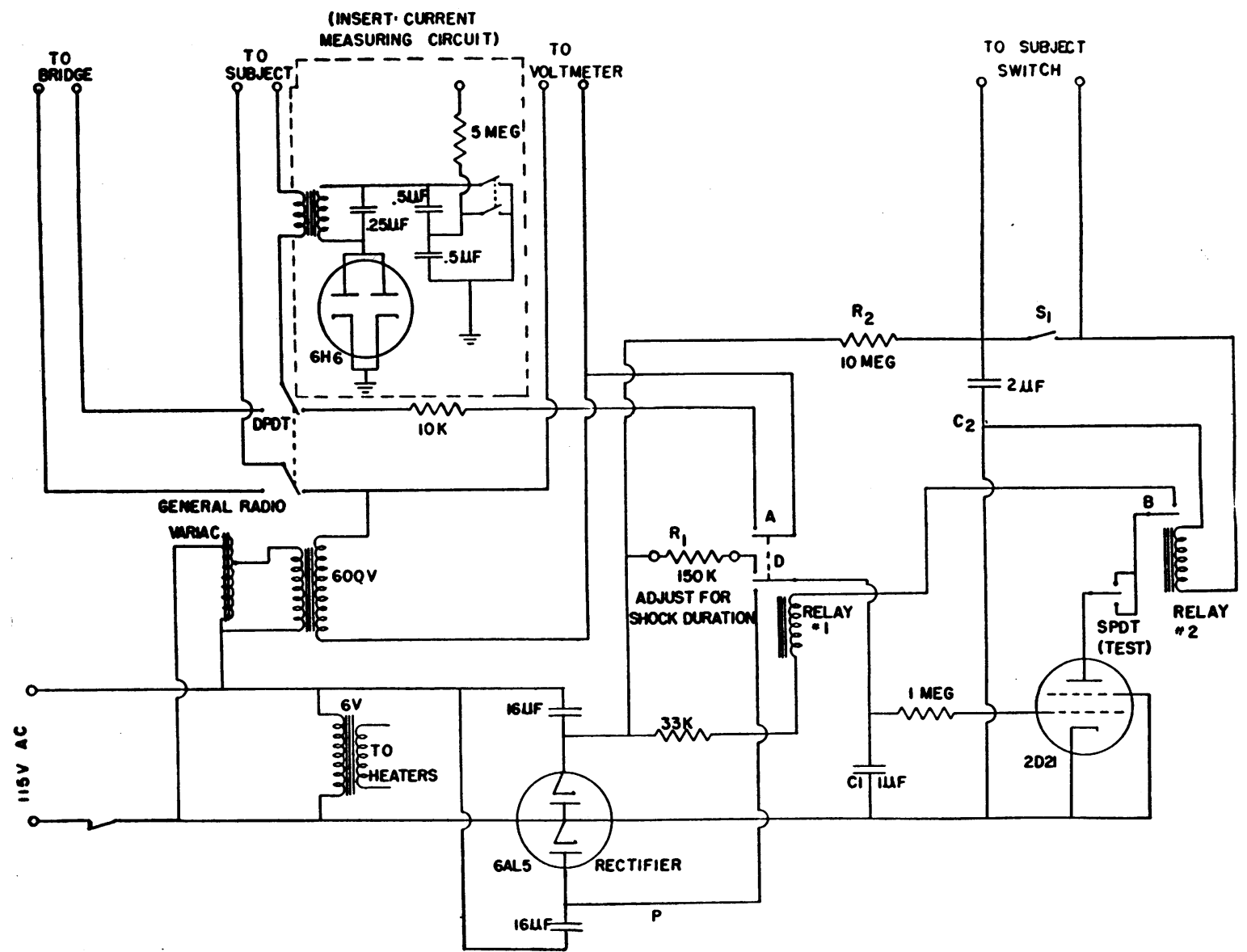

Fig. 1. Alternating Current Shocking Apparatus: 60 Cycle Strmulating Circuit, Stimulus Duration Control and Safety Devices

For explanation see text. 


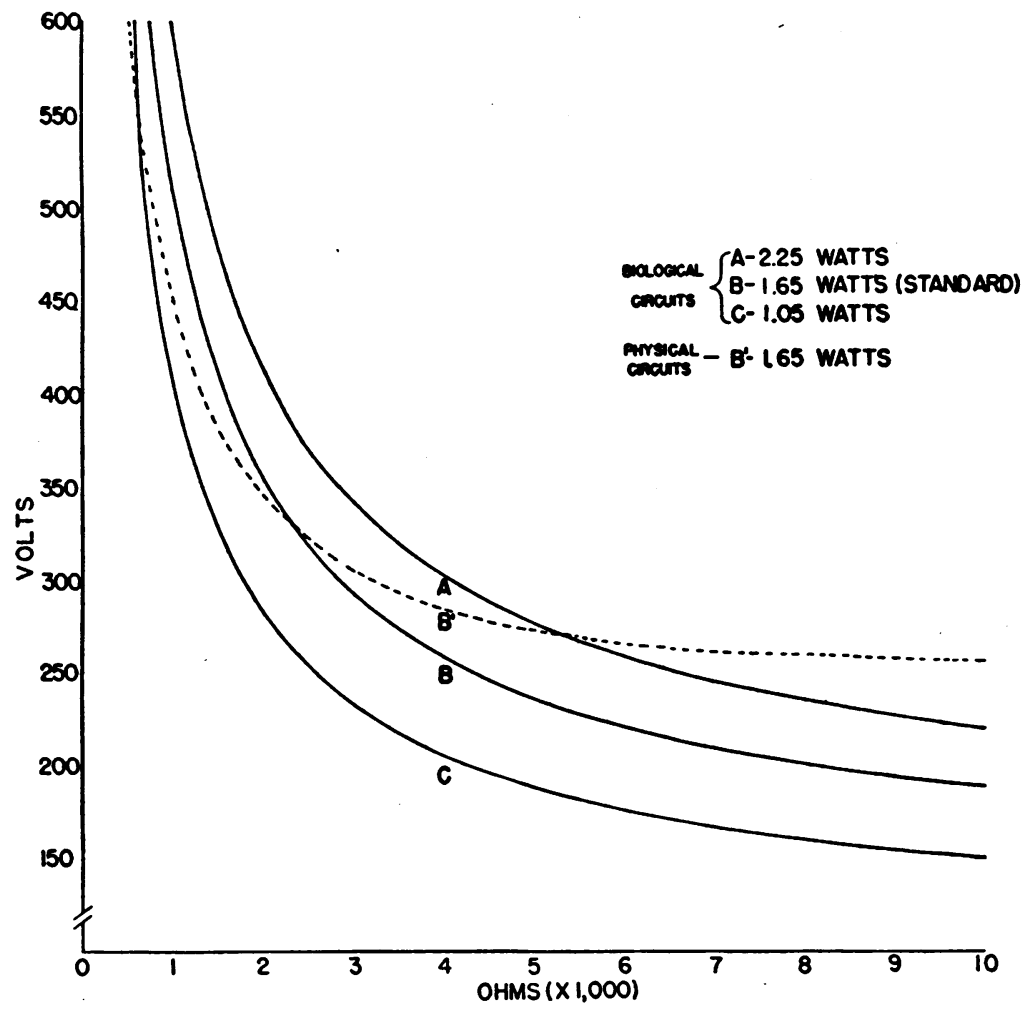

Fig. 2. Relationship between Voltage and Resistance for the Standard Power of 1.65 Watts in a Purely Physical Circuit (B'), and Similar Relationships for Several Biological Circuits

For explanation see text.

volunteered to serve as subjects for these experiments. Forty individuals were used in experiments which were made for the purpose of constructing empirical and rational power curves and 12 others served in experiments which were designed to determine whether electrically induced pain is related more closely to the amperage or the wattage of electric shock stimuli.

Procedure. A. Construction of empirical power curves. The following rather simple method was used for this purpose: (1) stimulating the experimenter and other "willing" subjects at progressively increasing intensities to a power level that was definitely painful; (2) selecting a group of subjects whose skin resistance would cover the range to be expected; (3) stimulating these subjects with powers that varied randomly, from those which were quite low to those which were considerably higher than the level which was painful to the experimenters; (4) plotting the delivered powers, from calculations of $\mathbf{P}=$ $I^{2} R$, as functions of skin resistance on the abscissa and voltage on the ordinate; (5) sketching a power curve covering all resistances, that was equal to the power reported as painful by the experimenters; (6) sketching two end power curves, one lower and one higher than the standard which were equidistant in power from the latter, and which could be distinguished from it in approximately 100 per cent of the trials; (7) interpolating, according to the calculated powers, four steps between each end point and the standard, thereby providing a scale of nine powers. (Only three curves are presented in Figure 2; these are for the standard and the two end powers.)

It was found that biological resistance, not including complemental resistance, ranged from approximately 700 to 10,000 ohms. For the reasons stated above, a complemental resistance of 10,000 ohms was added in series with the subject in the stimulating circuit. It will be noted, however, that biological resistance, or its equivalent, and not total resistance has been plotted in Figure 2. The addition of $10,000 \mathrm{ohms}$ of complemental resistance inverts the power curves from the position they would occupy were power calculated on the basis of the biological resistance alone as long as the biological resistance is less than the complemental resistance. (Simple calculations on the basis of Ohm's law will show that when the biological resistance is greater than the complemental resistance, the voltage which is necessary to deliver any given power to the subject increases concomitantly with increase in biological resistance.) Necessary voltage, on the ordinate, for delivering desired powers as these biological resistances, ranged from 150 to 600 volts. The power scale finally decided upon was built around a standard of 1.65 watts. These powers were approximately 1.05, 
$1.20,1.35,1.65$ (standard), $1.80,1.95,2.10$, and 2.25 watts.

In employing the charts for the purpose of delivering shock stimuli at any desired power, the appropriate power curve was identified and the necessary shocking voltage was determined for the particular skin resistance (complemental plus subject's resistance) before each stimulation.

B. Relationship of induced pain to characteristics of shock stimuli. The method for determining the relative effectiveness of amperage and power was rather simple after the apparatus and the empirical power curves were developed, and after it had been shown that skin resistance could be markedly reduced by scrubbing the fingers with saline paste. The 12 post-addict subjects were all given the same treatment and no medication was administered. After each subject was shown into the quiet, air-conditioned experimental room, the electrodes were applied and held in place by a $3 / 4$ inch rubber band. The electrodes consisted of two stainless steel plates measuring approximately $3 \times 5 \mathrm{~cm}$. which fitted the contour of the dorsal and palmar surfaces of the fingers. Each plate was connected to the stimulating apparatus by means of insulated wire leads which were fixed to binding posts in the center of the electrodes. They were coated with saline paste but were applied without scrubbing the fingers. Skin resistance was then obtained after balancing the capacitance. Under these conditions most individuals' skin resistance was found to be relatively high.

Six shocks of progressively increasing power were then delivered terminating with two of the standard stimuli of approximately 1.65 watts. ${ }^{2}$ To give the subject practice in comparing intensities, a complete series of nine shocks was administered in predetermined random order at approximately 45 -second intervals. The subject responded to each shock by reporting verbally whether it was stronger or weaker than the previously given standards. (No equal judgments were allowed.) On completing this practice series, the subject was given four more stimuli of standard strength, preceded by the following information: he was told to remember the strength of these standards since, after a one-hour interval, his judgment would be required on several more series. ${ }^{3}$ The subject was then returned to the waiting room.

2 We found frequently in our exploratory work that the first and sometimes the second stimulation produced greater power than predicted; thus during the initial stimulation for all individuals, the delivered power of each stimulus, including the first two standards, was calculated immediately after stimulation, using the formula $I^{2} R$. This procedure provided a check on the standard power, as it was occasionally necessary to shift the voltage upward or downward to obtain the desired power. Also, this procedure obviated excessive stimulation.

3 This method of administering the standards an hour previous to the experimental series was employed to parallel a subsequent discrimination study (14).
The subject returned to the experimental room after the one-hour interval, and the electrodes were re-applied. However, at this sitting, the subject's fingers were prepared somewhat differently. The fingers were cleaned with acetone and both surfaces were scrubbed thoroughly with a toothbrush coated with electrode paste. The lateral surfaces were wiped with acetone, and the electrodes applied with a uniformly thin covering of saline paste. Two test series, similar to the practice series except for differing stimulus order, were then administered. (The first stimulus for the first series was at a considerably lower power than the standard.) Prior to each of the series the subject was told to judge each shock as to whether it was stronger or weaker than the previously given standards, and not to compare them with each other.

The manipulation of skin resistance provided the pertinent data. Applying the electrodes without scrubbing the fingers previous to the practice series and administration of the four standards, resulted in standard shocks being given at relatively high and constant biological resistances. Thus, each individual received shocks of standard amperage and standard wattage. However, when the subject returned for the test series, the scrubbing procedure reduced the skin resistance markedly. The test series were power series; therefore, regardless of biological resistance, approximately the same powers were delivered that had been given during the practice series. But, due to the decrease in biological resistance, amperage, in contrast to power, was greatly increased over that given during application of the standards.

Thus, upon beginning the test series, the subjects had equal opportunity to judge on the basis of either amperage or power, since they had received standards of each, central tendencies being equal. As indicated, in order to make the first judgment decisive, a power much lower than the standard was administered as the first test stimulus. The power for this first stimulus was much lower than the standard, whereas, for the majority of subjects, amperage was much higher than the standard because their biologic impedance had been reduced greatly by the skin-scrubbing procedure. Hence, if the subjects judged the intensity of the stimulus on the basis of its power, they would respond "weaker," whereas, if judgments were made on the basis of amperage the response would be "stronger."

Treatment of data. Errors in discrimination were compared. Those that were made when calculations were based on amperage were compared with those made when calculations were based on wattage. The first comparison was between errors in judgment on the first stimulation of the first series; it was so clear-cut that no statistical tests of significance were required.

In that study it was necessary to determine whether morphine impaired the ability to discriminate different intensities of painful stimuli; therefore, all the pre-test standards were given under non-drug conditions and the one hour interval provided time for the administration and action of morphine. 
However, as one would expect, there were errors on both indices when a comparison of total errors was made for Series 1 and Series 2. These were evaluated by using the difference method of the $t$ test (Edwards [15]).

\section{RESULTS}

The functioning of the apparatus was satisfactory for our purposes. The mean of the delivered preparatory standards for 30 subjects was 1.66 watts, and the mean of the delivered series standards was 1.64 watts (predicted: 1.65 watts). The standard deviation of the differences between the preparatory and series standards for this group was 0.039 watts.

It was found, with one exception, that the voltage necessary to obtain a particular power in a biological circuit of a certain measured resistance does not correspond with the voltage calculated by means of Ohm's law. In Figure 2 are shown the relationships between $E$ and $\dot{R}$ in a purely physical circuit that includes only resistance (curve $\mathrm{B}^{\prime}$ ), and three different apparent powers in biological circuits (curves A, B, and C). ${ }^{4}$ The biological circuit included the subject's pre-stimulation resistance as well as unknown capacitance and inductance. It will be noted that for resistances that are considerably higher or lower than 2,300 ohms, the biological $E-R$ curve for the standard power diverges somewhat from the equivalent curve for physical power. In all probability this is because the effective $E$ for a biological circuit of unknown capacitance and inductance is a function of these variables and therefore is apt to be different from the pre-set $E$ which was estimated from calibrations on a purely physical circuit. Although changes in impedance following shock could not

'In Figure 2, curve $B^{\prime}$ represents the relationships between $E$ and $R$ for one given power (standard) as calculated from the equation $E=\sqrt{P R}$ which is another form of Ohm's law for a physical circuit. Curves A, B, and $C$ are rational curves for biological circuits derived from the equation $E=\sqrt{\frac{10 P(R+K)}{d}}$, in which $d=\frac{10 R}{K}$ $-\sqrt{\frac{R}{R+K}}$. These curves were found to correspond quite closely to the actual plots of observed $E$ and $R$ for any given power as calculated by the equation $P=I^{2} R$. Possible reasons for the differences in $E-R$ relationships in biological and physical circuits are discussed in the text. The use of the rational equation has the advantage that $P$ can be calculated in terms of $E$ and $R$ alone, without the use of $I$. However, curves identical with these would not be expected if the stimulator components were changed since the internal resistance and internal conductance would vary from instrument to instrument.
TABLE I

Significance for differences between the mean errors in judgment on amperage as opposed to those on wattage

\begin{tabular}{|c|c|c|c|c|c|c|}
\hline & \multicolumn{2}{|c|}{ Mean errors } & \multirow{2}{*}{$\begin{array}{c}\text { Mean } \\
\text { differ- } \\
\text { ence }\end{array}$} & \multirow{2}{*}{$\begin{array}{c}\text { Sigma } \\
\text { mean } \\
\text { differ- } \\
\text { ence }\end{array}$} & \multirow{2}{*}{$\mathbf{t}$} & \multirow{2}{*}{$\mathbf{P}^{*}$} \\
\hline & Power & Amperage & & & & \\
\hline $\begin{array}{l}\text { Series } 1 \\
\text { Series } 2\end{array}$ & $\begin{array}{l}2.08 \\
2.00\end{array}$ & $\begin{array}{l}4.42 \\
4.67\end{array}$ & $\begin{array}{l}2.34 \\
2.67\end{array}$ & $\begin{array}{l}0.742 \\
0.689\end{array}$ & $\begin{array}{l}3.15 \\
3.88\end{array}$ & $\begin{array}{l}<1 \% \\
<1 \%\end{array}$ \\
\hline
\end{tabular}

* The $P$ column indicates the percentage of such results that would be expected to occur by chance.

be measured, it was found that capacitance increases as biological resistance increases, being of the order of .05 microfarad at $10,000 \mathrm{ohms}$. It will be noted that the greatest divergence of the "physical" and "biological" curves occurred in the region at which pre-stimulation capacitance was greatest (Figure 2).

As would be expected, the $E$ to $I$ relationships for the biological circuits also did not conform to those which would be anticipated on the basis of Ohm's law in a physical circuit. The measured $I$ appeared to be largely independent of the prestimulation resistance, and was almost entirely a function of $E$. Therefore, as $R$ decreased, both $E$ and $I$ were increased for any particular delivered power.

The first treatment of the data of the experiments on the relationship of pain to the characteristics of shock stimuli was a comparison of errors for the first test stimulations. Scrubbing the fingers with saline paste before applying the electrodes for the test series resulted in a drop of skin resistance from a mean of 5,567 ohms for the preparatory standards to a mean of $1,758 \mathrm{ohms}$ for the first test stimulation. Mean power and mean current for these two conditions changed from 1.68 to 0.87 watts, and from 17.9 to $22.8 \mathrm{ma}$., respectively. Nine of the subjects received greater amperages on the first test stimulation than on the standard, while in all cases power was much less than the standard. All subjects who had' a clear-cut choice responded in favor of power, i.e., 100 per cent gave the response "weaker." This finding is all the more significant since, in many cases, the difference in amperage was much greater than indicated.

Table I shows the significance of the differences between total errors in judgment when all responses were evaluated in comparison with de- 
livered amperage or calculated power. The first comparison is for Series 1. Only one of the subjects made more errors when calculations were based on power than when they were based on amperage (this subject's judgments were quite erratic). The $t$ between power and amperage was 3.15. This probability of chance occurrence (less than 1 per cent), which is statistically very significant, would have been even less if a greater number of subjects had been tested or if more control of skin resistance had been obtained. ${ }^{5}$

Table I also presents a similar comparison for Series 2. The errors on amperage in relation to those on power were even more marked than for Series 1 ; the $t$ between errors was 3.88, giving a probability of chance occurrence of less than 1 per cent. The significance of this $t$ for Series 2 may be somewhat attenuated, however, since central tendency of judgments may have begun to operate.

\section{DISCUSSION}

The apparatus functioned satisfactorily in our experimental situation, and the errors between predicted and delivered power were comparatively small. Nevertheless, if much smaller step intervals of power were used, or if an experimental design necessitated great precision, further refinement in control would be needed. However, it would appear that the accuracy which was attained would be sufficient for the majority of psychological studies. Variability of several hundredths of a watt very probably would not be reflected in the outcome of conditioning work. Certainly for experiments in discrimination, and for other studies that we have underway in which short term disruption of behavior is introduced by shock, our control of power is far superior to control of voltage, or of amperage as previously used. ${ }^{\circ}$

\footnotetext{
- Several factors were operating against an outcome in favor of power: (1) as shown in a separate study (14), if the subjects were "anxious" they would tend to report stimuli as being "stronger"; (2) power was occasionally greater on first stimulations than predicted; (3) if the scrubbing procedure sensitized the skin there would be a tendency to report "stronger" more frequently.

- Although extrapolation may not be warranted from our results on verbal report to the effective stimulus in electroshock convulsions, investigations of controlled power in such situations might result in more adequate control of induced seizures. Arieff's paper (16) indicates some of the difficulties in this area.
}

Aside from comparatively small errors in predicting delivered power, there are several disadvantages in the present form of the apparatus. The complexity of design requires the services of either a physicist or a person of considerable electronic inclination. This complexity also makes it an impractical instrument for the average psychological or physiological laboratory in which severe limits are frequently placed upon expenditures. However, further work will probably result in a simplified method for controlling power.

As indicated by Curtis (13), it was maintained for some time that a completely new set of laws other than those describing physical circuits would have to be developed for assaying biological materials. However, as he points out, the apparent discrepancies may be quite easily due to the complexity of biological circuits, and to the great difficulty of compensating for, of controlling, or even of measuring changes in the material under study.?

As indicated, one of the sources of error may possibly be that of shock-induced changes in capacitance or in the other characteristics of impedance. In a circuit that includes resistance, capacitance, and inductance, power is expressed by the equation $\mathrm{P}=\mathrm{I}^{2} Z$. $Z$, the impedance of the circuit, is a function of resistance, capacitance, and inductance, the value of which can be determined only if the values of these variables and their series or parallel relationships are known. $\mathrm{Ob}$ viously, except for the subject's pre-stimulation resistance, such factors are unknown in the biological circuits obtained in the present experiment. Hence, in this paper the term "power" has been used to indicate the apparent power given by the equation $P=I^{2} R$. Although such a power index may not correspond in all instances with the true power, it has considerable utility in that its

Over 40 years ago, it was pointed out that in physiological studies, electric stimulators should be calibrated in terms of a particular physiological response (Martin, E. G., A quantitative study of faradic stimulation. I. The variable factors involved; and $V$. The influence of tissue resistance and of kathode surface on stimulating effectiveness. Am. J. Physiol., 1908, 22, 61; and 1910, 27, 226). This investigator demonstrated that the contractions of frog's gastrocnemius muscle correlated fairly well with the amperage of make or break faradic shock stimuli. He did not investigate the relationships between such responses and the wattage of electric shock stimuli. 
use makes possible the control of stimulation with respect to a physical measurement which, as has been shown, correlates highly with estimation of pain intensities. Actually, as mentioned, for resistances of about 2,300 ohms, "apparent power" corresponded quite closely with calculations of power based on Ohm's law in a purely physical circuit which included only resistance. In addition, the data show that power $\left(I^{2} R\right)$ may be predicted in a biological circuit with a comparatively small degree of error provided the pre-stimulation resistance of the subject is known and the initial impedance of the stimulating source is kept constant.

The main purpose of the experiment was to determine which characteristic of electric shock stimuli, amperage or power, was more closely related to the subject's report of the intensity of electrically induced pains. The two types of analysis of the data were in very close agreement. The data prove conclusively that power is the more important physical variable in determining verbal reports of the intensities of shock stimuli. In several studies mentioned in the introduction and in our own pilot work, evidence was adduced showing that voltage is a less significant variable than amperage. Therefore, we are led to the conclusion that of the three physical variables of shock stimuli, wattage is the one that should be controlled. When this is done, electric shock stimuli may prove to be not only a convenient stimulus for use in psychological studies on pain, but also an accurate one.

It should be pointed out that in our experiment the delivered powers of electric shock stimuli were nearly equivalent to delivered energies, since the duration of the stimulus was always 0.1 second. In this connection it is pertinent to note that the delivered energy of radiant heat stimuli has also proved to be useful in studies on pain (Hardy, Wolff, and Goodell [17]). This suggests the possibility that with any given technic for inducing pain, the relationship between pain intensity and the physical aspect of the stimulus can be described most accurately when the latter is expressed in terms of energy appropriate to its source-electrical, thermal, mechanical, photic, sonic, etc.

\section{SUMMARY}

1. An apparatus has been described that could be modified for controlling either the delivered wattage or the delivered amperage of electrical stimuli of fixed duration, and which provided a safety device to avoid accidental repetitive shocking.

2. With one exception, the data obtained indicated that the power developed by a particular voltage in a biological circuit of a certain measured resistance does not correspond with the power developed at the same physical resistance by the same voltage. This suggests that a biological circuit is characterized not only by resistance, but by unknown capacitance and inductance. Therefore, delivered wattages calculated on the basis of $\mathrm{P}=\mathrm{I}^{2} \mathrm{R}$, where $R$ is merely the pre-stimulation biological resistance, are only "apparent" powers. However, under the conditions of our experiments, such "apparent power" corresponded quite closely to $I^{2} R$ values calculated on the basis of an equivalent physical circuit.

3. Post-addict subjects judged the intensities of electric shocks delivered by the apparatus. The correspondence of the subject's verbal reports with the amperage and the wattage of stimuli was measured separately, and the differences were statistically evaluated. After a practice series which was followed by the application of four stimuli of standard amperage and wattage, resistances were reduced by scrubbing with saline paste the surfaces to which the electrodes were applied. Two consecutive power series of nine stimuli each were then administered. In each of these test series, one stimulus was of the standard power and four were higher and four were lower powers than the standard. Due to the decrease in resistance following the scrubbing procedure, the amperages of the test stimuli were considerably higher than those of the practice period. In response to each stimulus, the subject reported whether it was "stronger" or "weaker" than the previously administered standard.

4. It was shown quite conclusively that apparent delivered wattage correlates more highly than amperage with estimation of the intensities of electric shock stimuli.

5. The application of these findings to studies on pain and to other problems is discussed.

\section{ACKNOWLEDGMENT}

The authors wish to express their appreciation to $H$. $L$. Marks for his unstinted efforts in completing many of the tedious computations. 


\section{REFERENCES}

1. Tompkins, S. S., An analysis of the use of electric shock with human subjects. J. Psychol., 1943, 15, 285.

2. Lorge, I., Physiological aspects of lethal shock. Psychol. Bull., 1935, 32, 197.

3. Forbes, T. W., Muenzinger, K. F., and Wendt, G. R., Report of round-tables on the use of electric shock. Psychol. Bull., 1935, 32, 185.

4. Muenzinger, K. F., and Walz, F. C., An examination of electric current stabilizing devices for psychological experiments. J. Gen. Psychol., 1934, 10, 477.

5. Dalziel, C. F., and Lagen, J. B., Practical aspects of electric shock. Electrical West., 1941, March, p. 6 (Psychol. Abstracts, 1941, No. 2499).

6. Forbes, T. W., and Bernstein, A. L., The standardization of 60 cycle electrical shock for practical use in psychological experimentation. J. Gen. Psychol., 1935, $12,436$.

7. Gilmer, B. v. H., The sensitivity of the fingers to alternating electrical currents. Am. J. Psychol., 1937, 49, 444.

8. Jasper, H. H., A laboratory study of diagnostic indices of bilateral neuromuscular organization in stutterers and normal speakers. Psychol. Monog., 1932, 43, 72.

9. Kellogg, W. N., Electric shock as a motivating stim- ulus in conditioning experiments. J. Gen. Psychol., 1941, 25, 85.

10. Kellogg, W. N., Pronko, N. H., and Headlee, C. R., Continuous measurement of the effect of a depressing drug upon the organism. J. Comp. Psychol., 1942, 34, 195.

11. Spooner, A., and Kellogg, W. N., The backward conditioning curve. Am. J. Psychol., 1947, 60, 321.

12. Wikler, A., Sites and mechanism of action of morphine and related drugs in the central nervous system. Pharmacol. Rev., 1950, 2, 435.

13. Curtis, H. J., Bioelectric measurements, in Biophysical Research Methods, Uber, F. M., Editor. Interscience Publishers, Inc., New York, 1950, pp. 233-270.

14. Hill, H. E., Kornetsky, C. H., Flanary, H. G., and Wikler, A., The effect of anxiety and morphine on the discrimination of intensities of painful stimuli. J. Clin. Invest., 1952, 31, 473.

15. Edwards, A. L., Statistical Analysis for Students in Psychology and Education. Rinehart \& Co., New York, 1946, p. 174.

16. Arieff, A. J., Threshold studies in electrical convulsions with use of a square wave stimulator. Arch. Neurol. \& Psychiat., 1949, 62, 680.

17. Hardy, J. D., Wolff, H. G., and Goodell, H., Studies on pain. A new method for measuring pain threshold: observations on spatial summation of pain. J. Clin. Invest., 1940, 19, 649. 